\title{
ASO Visual Abstract: Pulmonary Metastasectomy in Patients with Lung Metastases from Nasopharyngeal Carcinoma
}

\author{
Ze-Rui Zhao, MD, $\mathrm{PhD}^{1,2}$, Di-Han Liu, $\mathrm{MD}^{3}$, Yi-Zhi Wang, $\mathrm{MD}^{1,2}$, Xue-Song Sun, $\mathrm{MD}^{4}$, and \\ Hao Long, MD, $\mathbf{P h D}^{1,2}$
}

${ }^{1}$ State Key Laboratory of Oncology in Southern China, Collaborative Innovation Center for Cancer Medicine, Department of Thoracic Surgery, Sun Yat-Sen University Cancer Center, Guangzhou, China; ${ }^{2}$ Lung Cancer Research Center, Sun YatSen University, Guangzhou, China; ${ }^{3}$ The Third Affiliated Hospital of Guangzhou Medical University, Guangzhou, China; ${ }^{4}$ State Key Laboratory of Oncology in Southern China, Collaborative Innovation Center for Cancer Medicine, Department of Nasopharyngeal Carcinoma, Sun Yat-Sen University Cancer Center, Guangzhou, China

The role of metastasectomy in nasopharyngeal carcinoma patients with lung-only metastasis remains unknown. This study (http://doi.org/10.1245/s10434-021-09597-5) found that the 5-year survival rate differed between nasopharyngeal carcinoma patients with and without pulmonary metastasectomy $(75.53 \%$ and $47.81 \%$, respectively). Hence, lung metastasectomy can be recommended in carefully selected patients.

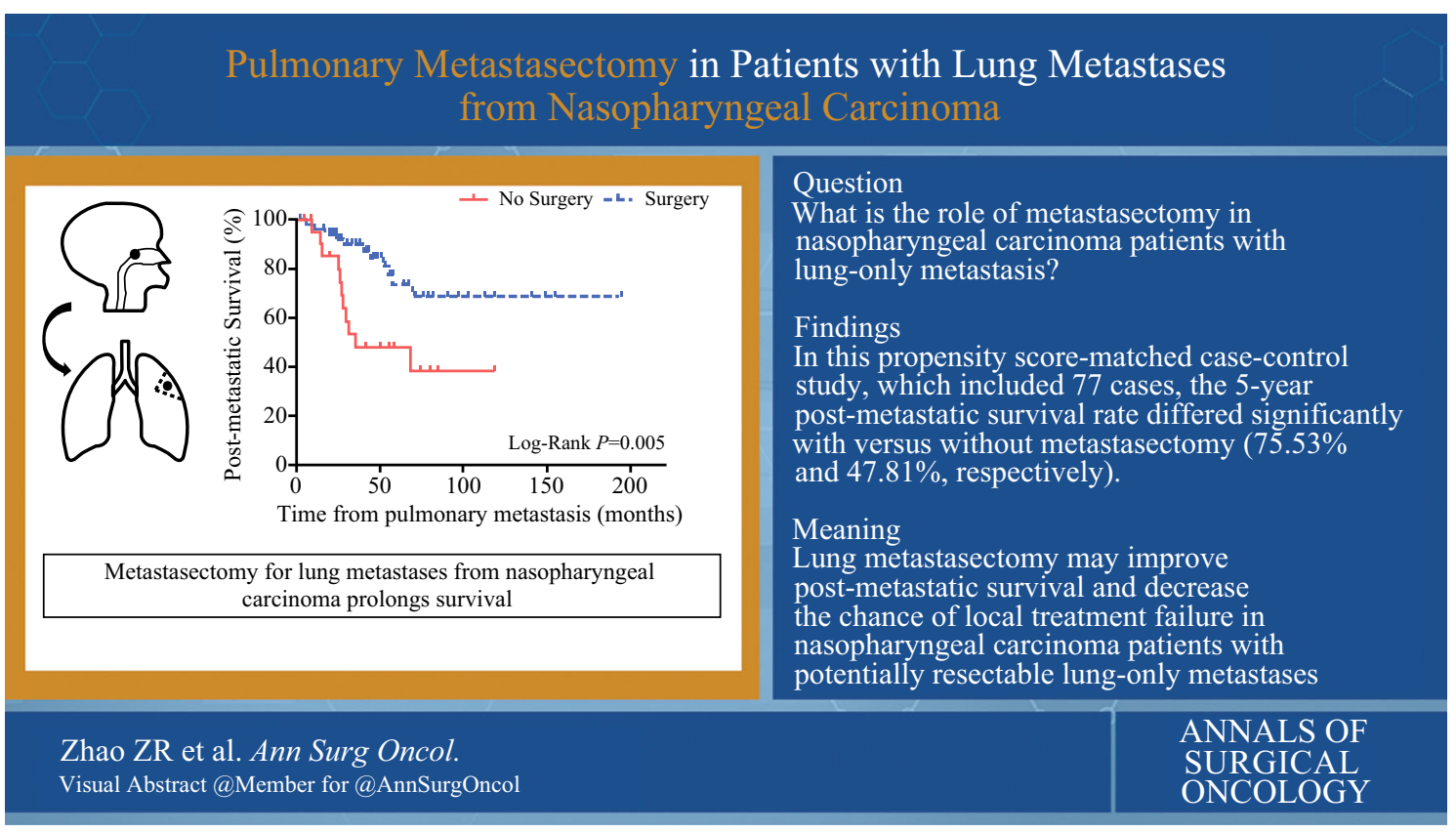

Ze-Rui Zhao, Di-Han Liu and Yi-Zhi Wang have contributed equally to this work.

(C) Society of Surgical Oncology 2021

First Received: 29 January 2021

Accepted: 29 January 2021;

Published Online: 17 April 2021

H. Long, MD, PhD

e-mail: longhao@sysucc.org.cn
DISCLOSURE None declared.

Publisher's Note Springer Nature remains neutral with regard to jurisdictional claims in published maps and institutional affiliations. 